\title{
Distributed System for Cognitive Stimulation Over Interactive TV
}

\author{
Carolina García Vázquez, Esther Moreno Martínez, Miguel Ángel Valero Duboy, and Ana Gómez Oliva
}

\begin{abstract}
This paper details the full design, implementation, and validation of an e-health service in order to improve the community health care services for patients with cognitive disorders. Specifically, the new service allows Parkinson's disease patients benefit from the possibility of doing cognitive stimulation therapy (CST) at home by using a familiar device such as a TV set. Its use instead of a PC could be a major advantage for some patients whose lack of familiarity with the use of a PC means that they can do therapy only in the presence of a therapist. For these patients this solution could bring about a great improvement in their autonomy. At the same time, this service provides therapists with the ability to conduct follow-up of therapy sessions via the web, benefiting from greater and easier control of the therapy exercises performed by patients and allowing them to customize new exercises in accordance with the particular needs of each patient. As a result, this kind of CST is considered to be a complement of other therapies oriented to the Parkinson patients. Furthermore, with small changes, the system could be useful for patients with a different cognitive disease such as Alzheimer's or mild cognitive impairment.
\end{abstract}

\section{INTRODUCTION}

$\mathbf{T}$ HE application of cognitive stimulation therapy or CST to the treatment of patients with Parkinson's disease (PD) is an evidence-based treatment, recommended for people in the initial stage of the disease, regardless of any drug treatments received. CST treatment usually involves repeated sessions of themed activities aimed to stimulate people with cognitive disturbances to improve their quality of life. CST groups can take place in different settings, including residential homes, hospitals, or day centers where therapists pinpoint, through the re- search and evaluation of each specific patient, exercises that are meaningful.

CST, like other health care services, can benefit from new low-cost electronic technologies such as web-enabled transactions, advanced networks, and new design approaches. However, developing new e-health services implies not only the application of new technologies, but also a change of healthcare processes based on the use of electronic communication and computer-based support at all levels and for all functions, both within the health care service itself and in dealings with outside suppliers [1].

In line with this approach, we have actively participated in the development and validation of an e-health service in order to improve the community health care services for patients with cognitive disorders. The work carried out by the authors is part of a larger program developed in cooperation with Spanish Parkinson's Associations to provide solutions aimed at helping people with Parkinson's disease receive CST. The solutions considered include the use of different devices for patients' interactions.

The new service makes the standard cognitive stimulation exercises suitable for interactive television (iTV) and it allows health care professionals to follow-up, via web, the therapies performed by them. The television set was chosen as the interaction device for Parkinson's patients because it is assumed that every home has a TV, the operation of which is familiar to all, making it easier for patients to accept such a new service.

In addition, from the outset, we consider that a therapist should intervene. When an exercise program is performed under the supervision of a therapist, there are more improvements in mental and emotional functions, daily living activities, and quality of life compared to a self-supervised exercise program at home [2]. Therefore, the exercises should be created dynamically, so that anyone, whether or not they are using the new service, could add exercises and customize them to patients' requirements. In addition, it is important to note that new e-health information service incorporates appropriate security measures, as the medical information exchanged by patients and therapists is sensitive in nature.

Through the use of this service, Parkinson's disease patients benefit from the possibility of doing therapy at home, using a familiar device such as a TV set, thus making visits to a specialized place unnecessary. Moreover, therapists benefit from greater control of therapy exercises performed by patients, allowing them to more easily supervise patients' results and customize new exercises according to the particular needs of each patient. However, because carrying out exercises in a group provides an optimal environment for learning and undeniable social benefits, therapy through iTV can also be administered in special places, such as Parkinson's Associations. 


\section{A. State of the Art}

There are presently several software initiatives to stimulate people with cognitive impairment. Such software can run on the PC (Smartbrain or MindFit), on game consoles (BrainTraining), on mobiles (Mobile Brain Trainer), or online (MyBrainTrainer). Many of these applications fall within the category of so-called "brain games," which have popularized brain training and brain stimulation. Most of these applications offer users a tool for cognitive stimulation exercises but they lack of any kind of interaction with professionals, who cannot supervise patients' evolution or participate in the design of the exercises.

Of the solutions available in the market specifically designed for structured neuropsychological evaluation and rehabilitation, noteworthy is the program Gradior PC, developed by the INTRAS Foundation [3]. This computer program provides a multimedia environment for professional brain training and rehabilitation of people with dementia, schizophrenia, cerebral palsy, mental retardation, and so on by means of a number of exercises: attention, perception, memory, calculation, language, and orientation. The therapist sets the characteristics and parameters of rehabilitation and the patient interacts with the computer through a touch screen. The cognitive activity to be performed is presented both visually and auditively. At the end of a session, the therapist can assess each patient's evolution and, according to the results, program exercises for the next session. The problem with this solution, however, is that exercises must be performed in the clinic or hospital where the computer is located and the therapist must be present to assist the patient in the use of the application. In addition, the application does not allow for dynamic incorporation of new exercises.

On the other hand, over the last few years, television has been used for care of disabled people at their homes due its almost universal presence and user-friendliness. Some examples of telehomecare include platforms such as ECG [4], which use videoconference systems and a standard TV set for patient interaction, allowing online remote monitoring of ECG, heart sound, and blood pressure.

From the technological point of view, the introduction of digital television allows the development of bidirectional interactive applications that use the Internet as a return channel. To date, the Multimedia Home Platform or MHP is the most widespread Java-based interactivity standard in Europe [5], [6]. MHP is the collective name for a compatible set of middleware specifications based on Globally Executable MHP, both defined by Digital Video Broadcasting. Currently, there are other initiatives in the market, mainly based on HTML with JavaScript, such as HbbTV (Hybrid Broadcast Broadband TV) [7]. The problem with this kind of alternatives is that, being based on HTML, they offer little dynamism.

Designed for care of disabled people in their homes, the OpenTDT project [8] in Spain provides access to social services such as cognitive stimulation and a reminder to take medication. However, this cognitive stimulation is used without monitoring by therapists and therefore with no evaluation of each individual patient. In the same way, Vital Mind [9] also enables CST using Digital TV but without therapists' participation.
A step toward the application of ICT in the field of cognitive stimulation is called "telecognitive stimulation." This technique can help people with cognitive impairment to receive stimulation sessions at home, providing a remote method of assessing impairment [10]. Thanks to ICT, systems can be accessible not only for patients but also for professionals who treat them. In this way, professionals can access the results of the exercises performed by patients and adjust the difficulty level to the each patient so as to minimize the evolution of the impairment. Furthermore, the results are stored for later analysis and assessment of the validity of treatment used.

As defined, no "telecognitive stimulation" service is presently available for patients with Parkinson's. This paper presents the full design, implementation, and validation of an e-health service designed to help people with Parkinson's disease maintain cognitive abilities. From the patient's point of view, its main innovation lies in its operability from home through of userfriendly devices such as a TV set and a remote control. It also makes therapist follow-up of patients easier, allowing for the customization of exercises in accordance with patient needs and easy incorporation in the system of any new exercises.

\section{MATERIALS AND METHODS}

\section{A. User Requirements}

The service was devised together with the therapist team of the Parkinson's Association of Madrid on the basis of standard therapy, and the process was undertaken on Association premises. The first step of the project was the definition of system requirements, for which a group of patients and therapists of the Parkinson's Association of Madrid (PKAM) was involved.

Patients' requirements were collected and the analysis results were as follows:

1) Patients need an application that will help them receive treatment. The technology used should be common in patients' life so as to avoid the digital divide. This application should also allow monitoring by the therapist.

2) The application should be usable and accessible by people with PD and people unfamiliar with technology; therefore, it should include the Design for All concepts.

3) The application should include time management to store the time that patients need to solve the exercises.

4) Due to motor problems of this disease, it is necessary to control the continuous clicks of patients using the TV remote control.

5) Information exchanged is considered sensitive for patients.

Similarly, therapists provided their requirements as follows.

1) The collections of exercises for cognitive stimulation used by the therapists should be adapted to the new environment.

2) Therapists should be able to monitor patients' therapy sessions, so a web interface for accessing data was proposed as the best solution. Such an interface would follow the criteria of the Web Accessibility Initiative [11] of W3C, using Web Content Accessibility Guidelines [12]. 


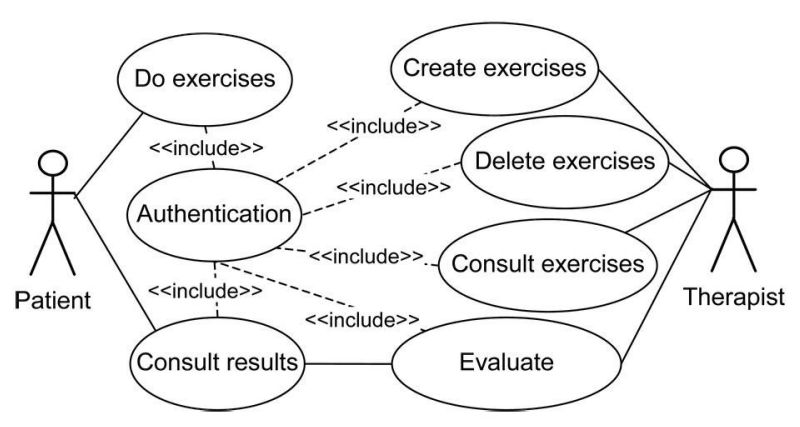

Fig. 1. Exercises' management case of use.

3) Access interface should be simple; it had to be designed for people that are unfamiliar with the use of technology in the process of patients monitoring.

4) The therapists should be allowed to introduce, delete, or modify the stored exercises in an intuitive way, without the assistance of a programmer.

5) Definition is required of an evaluation framework to allow patients and therapists to validate the final system.

Once these requirements had been collected, we proceeded to perform the analysis and system design. This study was carried out in cooperation with the same team of therapists of Parkinson's Association of Madrid.

\section{B. System Design}

The main scenario of this system is Exercises' management (shown in Fig. 1) where the patients can solve a collection of exercises and afterward see the evaluation given by the therapist. The therapist evaluates the patients' exercises and can create new exercises by either deleting the old ones, or by choosing exercises from the store. In addition to the foregoing scenario, other possible scenarios are as follows.

1) Appointment management: the therapist updates the next appointment at PKAM Association for the purpose of informing the patients.

2) Users' management: to carry out tasks of inserting or deleting user profiles in the system.

The results of each patient's exercises are stored in a database and later analyzed by the assigned therapist via the web. Depending on the results, the therapist may change the level of difficulty for the next collection of exercises or insert a comment about these results. These comments may be viewed by the patient through the interactive TV application.

\section{Therapy Design}

One of the biggest challenges was to translate the exercises on paper to digital format [13] to send them electronically to patient's equipment. A workgroup formed by engineers of the Universidad Politécnica de Madrid (UPM) and therapists from PKAM Association analyzed the different kinds of recommended exercises for CST, looking for a pattern that would allow them to define a common digital format for all of them. Then, all the previous exercise books were adapted to be used in digital TV.

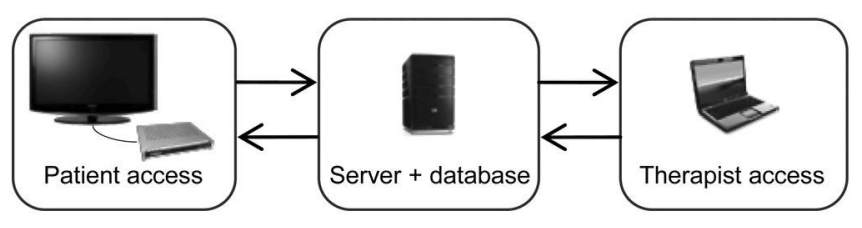

Fig. 2. System architecture.

Moreover, these exercises tested patients' capabilities such as attention, initiative, categorization, planning, arithmetic, and memory. Therefore, different collections had to be designed in order to provide these exercises through the system.

The therapy was designed by grouping the exercises in collections of nine, each belonging to a different stimulation area. PKAM therapists thought it would be better for patients to solve an exercise in each area in a single session to avoid boredom.

These collections are classified at different levels of difficulty. First, the patient downloads the easiest collection and the therapist evaluates the results and changes the level of difficulty of the collection for the next session, if necessary.

\section{Implementation}

The introduction of iTV along with a set-top box at users' homes opens up a new range of possibilities for the development of applications that can interact with remote machines using the Internet as a return channel. There are two different technologies in this system: one is on client side and the other is on the server side. The client side uses the standard MHP that enables the reception and execution of interactive Java-based applications on the set-top boxes in patients' homes. The MHP version used is 1.1 .2 , which permits interaction with a server via a return channel. For this development, the tool employed was iDesigner [14], from Mit-Xperts Corporation. The server side is based on Java platform, using JSP that interacts with MySQL database through Java core.

As shown in Fig. 2, the architecture of this system includes the following four devices.

1) TV set + set-top box (STB): this is the equipment patients need to carry out the CST. A TV set is a conventional device and the STB is a DTT decoder which permits the access to CST.

2) Personal computer: used by therapists to access the patient answers for evaluation.

3) Server: used for storing personal data of the patients and also to decode the messages or Protocol Data Units (PDUs) from the STB and to send the appropriate responses.

For the connection between the patient's STB and the server, we designed an ad hoc protocol, with a view to minimizing data exchange in order to enhance the speed of the system.

All the messages from STB to server include the user identifier. Furthermore, messages containing the answers from the patient incorporate the time spent on each exercise.

On the server side, messages contain the information requested by the STB, such as the name and gender of the patient, the therapy exercise collection, the evaluation results, the 


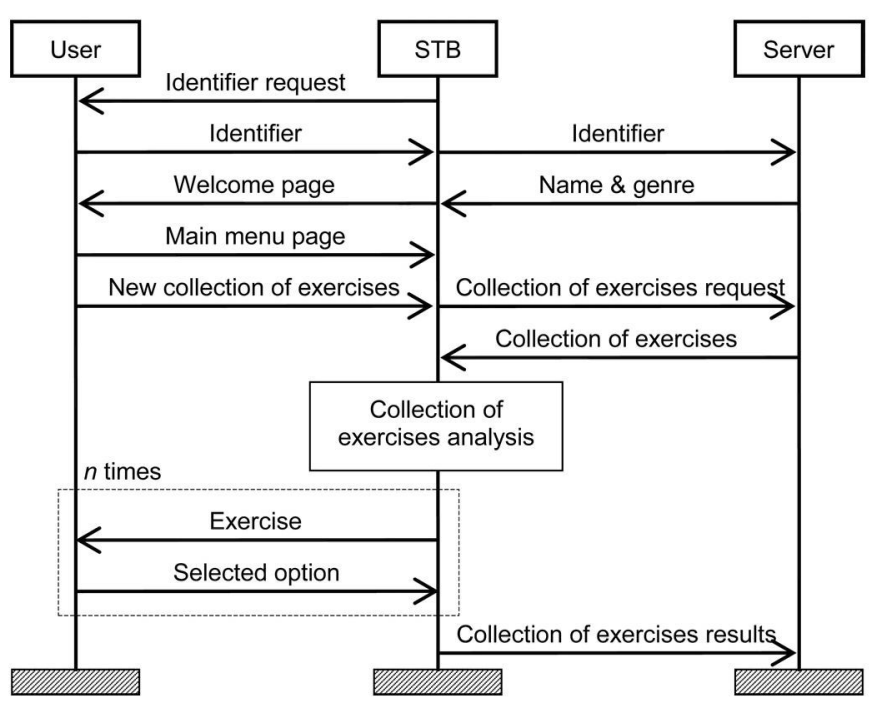

Fig. 3. Sequence diagram of the information exchange.

appointment for the next session, or a possible error message. The most interesting message is the one that includes the exercise collection. Here, each exercise is described by its type, statement and time limit; there is also an associated image, answer values (strings or pictures in byte-array format) and the number of the right answer.

Fig. 3 describes the sequence diagram of the information exchange between a patient, the STB installed at patient's home and the remote server. First, the patient introduces his identifier on the application. Next, if patient selects New collection of exercises in the main menu page a new collection of exercises, dynamically created, will be sent to the STB. When the patient finishes it, results are sent to the server for therapist evaluation.

\section{E. System Security}

The different devices of the platform exchange medical information that is very sensitive. The communication between the application run in the STB and the server is highly confidential [15] because if medical information is compromised, this might jeopardize a patient's life. Therefore, the designers need to secure the data and the communication media between the users and the server to protect patients' privacy and enhance the delivery of health services.

Accordingly, the designers have incorporated appropriate security mechanisms in the information exchange, using the SSL protocol to safeguard confidentiality and to provide mutual authentication.

\section{F. Graphic User Interface}

The design of user interfaces fulfills the requirements of usability, accessibility, and ubiquity necessary for Parkinson's patients.

The patient using the remote control can control the TV application through an interface with a high degree of usability. In addition, the navigation diagram of the application is very simple and the menus are only two levels deep.
In order to make it easier for therapists to use the platform, all pages include a link to the particular help page.

Fig. 4 shows some images of screens of the patient application. In particular, they are examples of three different types of exercises.

1) Exercises types 1 and 2 show a statement with three possible answers where the patient has to select just one. The difference between types 1 and 2 is that in the first case the answers are strings and in the second one, pictures.

2) Exercise type 3 displays a statement and some pictures and the patient must write the answer using the keyboard on the remote control.

All exercises are created in the same page of MHP application. This design criterion has many advantages. One of them is that the MHP application is smaller, which is a very useful feature if the application is associated with a TV channel due to the limited available bandwidth. On the other hand, the system makes it easy for people who do not normally use technology to create new exercises. This means that is not necessary to turn to a programmer to add new exercises to the cognitive stimulation database.

The evaluation of the patient answers selected is not fully automatic because therapists prefer to check the results personally. Fig. 5 illustrates how the therapist evaluates the exercise collection the patient has finished. The screen is divided into three parts. The first part of the page shows a list of the already evaluated exercise collections. The second part describes the downloaded collections that the patient has not yet finished. The last part presents the collection of patient answers for evaluation, including the identifier of the collection, the download and finishing dates, and the situation of each exercise with a tic, a cross or a clock if the answer is right, wrong, or out of time, respectively. In addition, in the two first cases, the time spent on each exercise is shown. Finally, there is a space for the therapist to add comments and indicate the level of difficulty of the next collection of exercises.

Finally, we have developed the platform so as to allow addition of new exercises to the database. Once again, the main design criterion was that this process should be easy for people not familiar with technology. According to the type of exercise, the therapist completes the cognitive area which is stimulated by this exercise, level of difficulty, description and statement, type of exercise, and time limit to finish it. On the second page, the therapist can confirm the data previously introduced and then select the correct answer for automatic validation, either by writing the text or by looking for a particular picture in the associated pictures database.

\section{Evaluation and Results}

\section{A. Evaluation Plan}

The evaluation plan was designed by the workgroup formed by therapists and engineers, according to the following process.

1) First, UPM engineers performed functional validation in the laboratory with the support of a therapist.

2) Next, the workgroup designed two types of surveys: one for patients and one for therapists. The two kinds of 

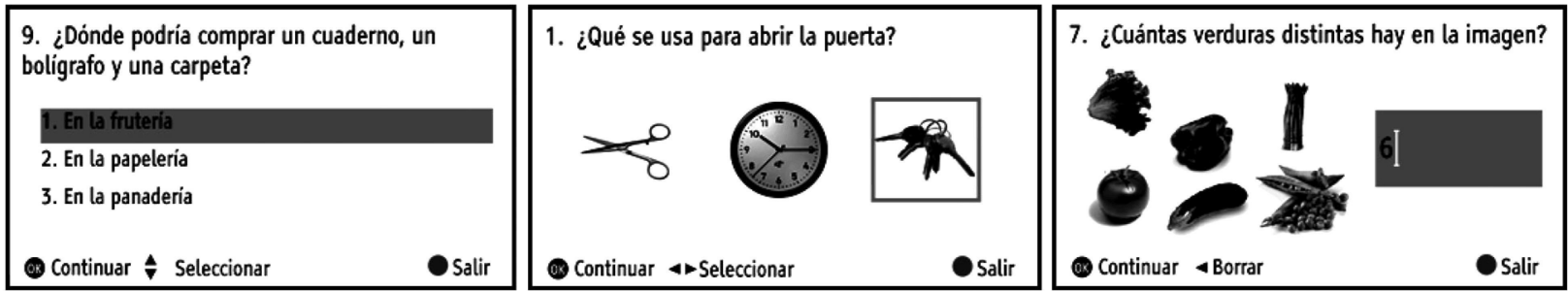

Fig. 4. Exercises types 1, 2, and 3, respectively.

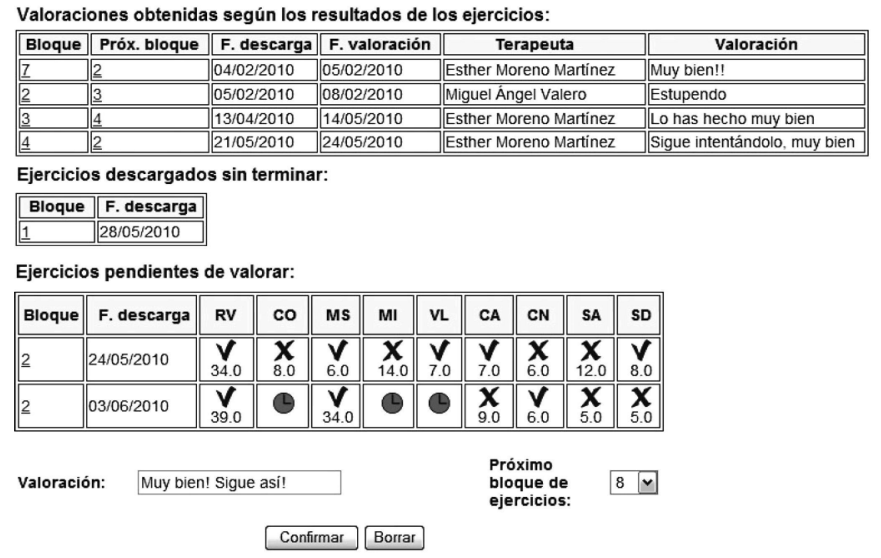

Fig. 5. Therapist page with patient progress.

surveys evaluated the system's accessibility and usability. Furthermore, this process aimed to determine whether it might be feasible to provide patient therapy at home. Section D presents the results obtained.

3) Finally, the experience was carried out at PKAM Association with patients previously selected by the therapeutic team.

\section{B. Patient's Requirements}

The workgroup selected patients to receive therapy with this system according to the following requirements.

1) Patients could not be in advanced state of PD. Therapists selected patients with lower than GDS 5 because they are the intended recipients of this kind of therapy [16].

2) Patients need not be habitual users of technology.

3) There should be parity between men and women.

4) The study should include the widest possible age range.

Then, the PKAM therapist selected patients to participate in the experience.

\section{Users' Sample}

Finally, the patient sample was the next.

1) 17 patients, with 11 men, and 6 women.

2) The average age was 60 years; the youngest was 44 and the eldest was 83 .

3) In general, the patients had a low-to-medium level of access to technology.
TABLE I

PATIENT'S RESULTS

\begin{tabular}{|c|c|c|c|c|c|}
\hline Question & TA & $\mathrm{A}$ & $\mathrm{I}$ & $\mathrm{D}$ & TD \\
\hline $\begin{array}{l}\text { I easily performed cognitive } \\
\text { stimulation exercise using remote } \\
\text { control and the TV }\end{array}$ & 8 & 9 & 0 & 0 & 0 \\
\hline $\begin{array}{l}\text { I think having my cognitive } \\
\text { stimulation therapy using the TV } \\
\text { is fun }\end{array}$ & 8 & 7 & 2 & 0 & 0 \\
\hline My exercises were easy & 16 & 1 & 0 & 0 & 0 \\
\hline $\begin{array}{l}\text { The application response time is } \\
\text { good }\end{array}$ & 0 & 2 & 6 & 4 & 5 \\
\hline $\begin{array}{l}\text { It is useful to be able to check my } \\
\text { next appointment with my } \\
\text { therapist }\end{array}$ & 6 & 6 & 5 & 0 & 0 \\
\hline $\begin{array}{l}\text { It is good to be able to see the } \\
\text { therapist's evaluation of my } \\
\text { results }\end{array}$ & 8 & 8 & 1 & 0 & 0 \\
\hline $\begin{array}{l}\text { I like having my cognitive } \\
\text { stimulation therapy using TV } \\
\text { remote control }\end{array}$ & 8 & 6 & 3 & 0 & 0 \\
\hline $\begin{array}{l}\text { If I could, I would have my } \\
\text { therapy using TV at home }\end{array}$ & 6 & 7 & 3 & 1 & 0 \\
\hline
\end{tabular}

Three therapists participated in this experience. One is a neuropsychologist, the other a speech therapist and the third a social worker.

\section{Results}

UPM engineers installed a TV and the application on STB MHP 1.1.2 at the PKAM Association and the patients and therapists used it for a year. The therapists had a PC for accessing the web platform and monitoring patients' results.

Table I shows the results of patient surveys: totally agree (TA), agree (A), indifferent (I), disagree (D), or totally disagree (TD).

The results of experience were considered highly positive. To improve the system, patients suggested increasing exercises' difficulty and providing higher quality images. Other suggestions called for improving the efficiency of the application and providing the option of a keyboard on the TV screen to make typing easier. What follows is a summary of opinions from patient surveys.

1) They love having interactive therapy because, for some of them, it is easier and faster to use the remote TV control than to write on paper, especially for people who use mobile phones.

2) They are pleased to have the option of working through the TV, but without giving up group therapy.

3) They like having therapy at home. 
4) In general, they like having part of their therapy in this way.

On the other hand, the therapist team emphasizes that the experience was highly positive because patients like training with this platform. From their point of view, the system is a useful tool in their daily work, providing more exercises at different levels, thus, reaching all the profiles of PD patients.

\section{CONCLusion AND Future Works}

The main conclusion of this study is that CST through digital TV is possible because patients are familiar with the TV in their daily life. In addition, the patients' application has been developed to be easy to use, with a simplified remote control and accessible and useful interface. Thus, we believe that the use of TV set instead a PC could be a major advantage for some patients whose lack of familiarity with the use of a PC means that they can do therapy only in the presence of a therapist. Therefore, the use of an alternative device as TV could bring about a great improvement in their autonomy.

The therapist team has made a positive assessment of the web platform for monitoring patients and exercise management. The use of this platform allows for more personalized therapy because the therapist can easily change the therapy according to patient results. In addition, it enables deeper and quicker knowledge of the patient and his or her progress. Therefore, the patient receives better care by performing specially designed collections of exercises.

Moreover, this therapy demands no extra time from the therapist. In a traditional therapy session, patients have the therapy in groups of five people and then the therapist evaluates their results. The service makes evaluation easier for therapists.

In conclusion, such a system allows for the better evaluation of the situation of patients with PD. In general, both patients and therapists greatly appreciate this therapeutic approach, and the ability to have it at home enhances the group therapy at Parkinson's Associations.

As a result, this kind of CST is considered to be a complement of other therapies oriented to the Parkinson patients. Furthermore, with small changes, the system could be useful for patients with a different cognitive disease such as Alzheimer's or mild cognitive impairment. The authors are aware that more patients and therapists must be involved with having this kind of therapy for a longer period. This would contribute to improving the efficiency and effectiveness of the service.

This study is oriented to develop this service on other Javabased platforms. We have migrated the service and developed an Android 3.0 application also based on Java technology. This development will, in the near future, allow for therapy with patients through a tablet. Availability of a portable device that can be used anywhere will unquestionably result in better conditions of service for patients. This application is going to be tested in two Spanish Parkinson's Associations to evaluate its usefulness. Future work includes the use of artificial intelligence techniques for automatic evaluation of the exercise collections.

\section{ACKNOWLEDGMENT}

The authors wish to thank the Parkinson's Association of Madrid for its collaboration in all phases of development.

\section{REFERENCES}

[1] R. Richardson, S. Schug, M. Bywater and D. Lloyd-Williams, "Development of eHealth in Europe. Position Paper," EHTEL Thematic Working Group eHealth (T2) under the auspices of EC-founded project PROEHTEL (IST-1999-29029), European Health Telematics Association, p. 3, Jan. 2002, re-edited in Apr. 2004. [Online]. Available: http://www.ehtel.org/publications/publications-ehtel-reportsand-papers/PROEHTEL-Del-08-T2.1-format-new-electronic-2004-0419.pdf/at_download/file.

[2] E. E. Dereli and A. Yaliman, "Comparison of the effects of a physiotherapist-supervised exercise programme and a self-supervised exercise programme on quality of life in patients with Parkinson's disease," Clin Rehabil., vol. 24, no. 4, pp. 352-362, Apr. 2010.

[3] M. A. Franco, Y. Bueno, T. Cid, and T. Orihuela, (Apr. 10, 2012), "Programa GRADIOR: Rehabilitación cognitiva por ordenador" Fundación INTRAS. [Online]. Available: http://psicologiaonline.com/ciopa2001/actividades/09/index.html

[4] S. Guillén, M. T. Arredondo, V. Traver, J. M. García, and C. Fernández, "Multimedia telehomecare system using standard TV Set," IEEE Trans. Biomed. Eng., vol. 49, no. 12, part 1, pp. 1431-1437, 2002.

[5] J. Clover, (Dec. 2, 2011), "EBU commits to hybrid future," Broadband TV news. [Online]. Available: http://www.broadbandtvnews. com/2011/12/02/ebu-commits-to-hybrid-future/

[6] Spanish Commission of Telecommunications Market. "Annual Rep.," 2009.

[7] HbbTV Consortium, (Aug. 27, 2009). "New European initiative merges television with the power of the Internet," [Online]. Available: http://www.hbbtv.org/pages/news_events/pdf/HBBTV_PR_Final_ 20090827.pdf

[8] OpenTDT Project, Main page. (Apr. 10, 2012). [Online]. Available: http://www.opentdt.com

[9] A. Miotto, J. Lessiter, and J. Freeman, "Vital mind: An interactive set-top box platform for cognitive training applications," in Proc. Virtual Rehabil. Int. Conf., 2009, p. 207.

[10] F. Díaz, M. A. Villanueva, A. Balo, A. López, A. I. Pedreira and J .C. Millán-Calenti, "TeleGerontología: Un nuevo recurso de apoyo gerontológico a domicilio," Tékhne. Revista de Estudios Politécnicos, vol. III, no. 5/6, pp. 57-71, Jun. 2006.

[11] World Wide Web Consortium. (11 Oct. 2002). "Implementation plan for web accessibility," Web Accessibility Initiative. [Online]. Available: http://www.w3.org/WAI/impl/Overview

[12] World Wide Web Consoritum, (Dec. 11, 2008). "Web Content Accessibility Guidelines 2.0," W3C Recomendation. [Online]. Available: http://www.w3.org/TR/WCAG20/

[13] Parkinson's Association of Madrid, "Cuaderno de ejercicios," Consejos Sobre Trastornos Cognitivos Para Pacientes Con Enfermedad de Parkinson, Biblioteca Parkinson, Madrid, Spain, 2007.

[14] Mit-Xperts Corporation (Apr. 10, 2012). "Mit-Xperts iDesigner," Products information. [Online]. Available: http://www.mit-xperts.com/ products/idesigner/

[15] R. Sulaiman, D. Sharma, W. Ma, and D. Tran, "A security architecture for e-health services," in Proc. 10th Int. Conf. Adv. Commun. Technol., 2008, vol. 2, pp. 999-1004.

[16] B. Reisberg, S. H. Ferris, M. J. de León, and T. Crook. "The global deterioration scale for assessment of primary degenerative dementia," Amer. J. Psychiatry, vol. 139, pp. 1136-1139, 1982. 